\title{
The Hollow Fiber Infection Model for Antimicrobial Pharmacodynamics and Pharmacokinetics
}

John J.S. Cadwell*

Fiber Cell Systems Inc. 905 West $7^{\text {th }}$ Street, \#334, Frederick, Md. 21701, USA

\begin{abstract}
The recent introduction of hollow fiber bioreactor cartridges represents an important advancement in the field of in vitro toxicology. Many toxicologists believe in vitro testing methods are a useful, time and cost effective tool for drug discovery but it is generally accepted that many of the available tests are not effective for examining both time and concentration, and do not closely mimic human kinetics. This is because they do not properly take into account pharmacodynamic actions (what a drug does to the body) and pharmacokinetic actions (what a body does to the drug). Recently, with the use of hollow fiber bioreactor cartridges, this has changed. The hollow fiber infection model is a useful addition to standard in vitro toxicology methods as it mimics changes in drug concentration over time, as they would occur in humans. An overview of historic $\mathrm{pk} / \mathrm{pd}$ models is presented and the utility of the hollow fiber infection model as it relates to antibiotics and other drugs are discussed.
\end{abstract}

Keywords: Hollow fiber infection model; Antibiotic resistance; Antibiotics; In vitro; Pharmacodynamics; Pharmacokinetics

\section{Introduction}

$\mathrm{Pk} / \mathrm{pd}$ assays are one of the first steps in determining the potential utility of a specific compound for antimicrobial activity. Efficacy and safety at specific dosages must be determined and potential side effects need to be identified. Over the years many assays have been developed which control the exposure of a target bacteria to a specific concentration of an antibiotic. These assays can use animal models or be in vitro. In vitro models can be defined by the number of variables that are controlled (time and/or concentration) and whether the target bacterium is retained within a compartment or not. Compartment models are defined as either having one compartment or two compartments. In the past decade the two compartment hollow fiber infection model has emerged as a potentially useful adjunct to other in vitro assays.

Pharmacokinetic-pharmacodynamic (PK-PD) principles for antibiotics were initially identified in the 1940s and 1950s by Dr. Harry Eagle [1]. Using animal models he identified the time dependence of penicillin bactericidal activity, the concentration dependence of streptomycin and bacitracin activity and the mixed pattern for tetracyclines. These data were applied to the clinic where it was found that continuous infusion of penicillin resulted in the most rapid cures while avoiding drug related toxicities. The most effective dosage regimens for concentration dependant antibiotics like streptomycin were those that resulted in the highest initial peak concentrations. Out of this work were derived the first principles of antibiotic action, that both time and concentration plays an important role in antibiotic effectiveness (Figure 1).

In the case of antibiotic development, it is critical to define both the time and concentration interactions of the drug with the target organisms as early as possible. The drug's spectrum of activity and potency against different strains of bacteria also needs to be determined. Traditional assays for susceptibility include the disc test, the E-test and broth dilution assay. These provide a quantitative measurement of the drug's potency and are defined as the minimum inhibitory concentration or MIC. The MIC is the lowest concentration of the drug that prevents a bacterial inoculum from growing to visibly detectable levels. The three most common PK-PD measures derived from the
MIC are the duration of time a drug concentration remains above the MIC, the ratio of the maximal drug concentration to the MIC and the ratio of the area under the concentration time curve at 24 hours to the MIC. MIC is the first and most important specification for an antibiotic that helps to define its therapeutic potential. However the MIC tells us nothing about:

\section{1) Whether an antibiotic is bacteriostatic or bactericidal}

2) Whether this activity is time dependant or dosage dependant

3) The rate of bacterial killing

4) Whether the drug exhibits a post-antibiotic effect when it falls below the MIC

5) What parameters of drug exposure most influence efficacy

6) The most effective pharmacodynamic targets for optimal dosing and

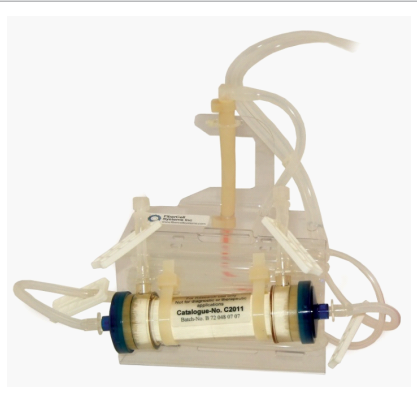

Figure 1: Hollow fiber bioreactor.

*Corresponding author: John J.S. Cadwell, President and CEO of Fiber Cell Systems Inc. 905 West $7^{\text {th }}$ Street, \#334, Frederick, Md. 21701, USA, E-mail: jjcadwell@comcast.net

Received December 07, 2012; Accepted January 03, 2013; Published January 05, 2013

Citation: Cadwell JJS (2012) The Hollow Fiber Infection Model for Antimicrobial Pharmacodynamics and Pharmacokinetics. Adv Pharmacoepidem Drug Safety S1:007. doi:10.4172/2167-1052.S1-007

Copyright: (C 2012 Cadwell JJS. This is an open-access article distributed under the terms of the Creative Commons Attribution License, which permits unrestricted use, distribution, and reproduction in any medium, provided the original author and source are credited. 
7) Which dosage profiles either prevent or facilitate the development of resistance.

A time related assay capable mimicking human antibiotic pharmacokinetics on a population of organisms large enough to reveal the emergence of resistance is required [2].

\section{No Variables (Time and Concentration are Fixed)}

Current methods for performing in vitro static assays for toxicological results and efficacy are relatively historic in nature. They consist of discs of paper soaked in differing concentrations of the drug in question placed onto petri dishes containing agar and the target organism. The size of the ring of inhibition of bacteria growth can be used to calculate the minimum inhibitory concentration. The E-test is a refinement of this assay where a plastic strip is coated with a gradient of the drug and then placed onto agar containing the target bacteria. There are two types of dilution assays that can be used to determine the MIC of a particular antibiotic. They are the broth macrodilution assay and the broth microdilution assay. The difference between these two assays is the volume of broth used and how the assay can be quantified. For the macrodilution assay $15 \mathrm{ml}$ test tubes are used and the antibiotic is serially diluted over a wide range. The MIC is determined by the visual detection of bacterial growth inhibition. The microdilution assay is performed in a similar manner but a microtiter plate is used instead of test tubes and the volumes are typically at least $100 \mathrm{x}$ smaller. The use of microtiter plates allows quantitation to be easily performed in an automatic plate reader (Figure 2).

\section{One Variable (Time is Variable, Concentration is Fixed)}

Advancement over the MIC assay is the static time kill assay. In this assay cultures of bacteria are exposed to static concentrations of an antibacterial agent over a defined period of time. Unlike the MIC assay, samples are taken at intervals of time and actual counts of viability are taken. The static kill time assay does provide some information about the rate of efficacy but the static concentrations of antibacterial agents are still lacking in clinical relevancy.

\section{Two Variables (Time and Concentration are Both Variables)}

\section{Animal models of infection}

In vitro pharmacodynamic models, MIC and static time-kill assays provide good methods for examining pharmacodynamics. They lack the complex interactions between host and drug that is present in animal and eventually human clinical models. Some of the advantages of animal models include 1) the ability to evaluate clinical efficacy in addition to antibacterial pharmacodynamics 2) examination of the

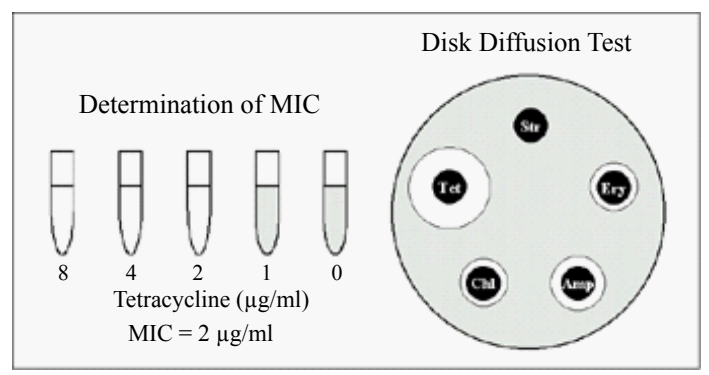

Figure 2: Examples of methods for the determination of minimum inhibitory concentration. interaction between drug therapy and host immune response and 3) ability to examine the interaction of drug with serum proteins. However, there are also problems associated with animal models. It is expensive to maintain animal colonies and time consuming to perform the experiments. Many infections simply cannot be reproduced in animals. Experimental design can be subject to regulatory approval and the experiments need to be carried out in a humane fashion. Drug kinetics may be very different than that found in humans. The total bacterial load is generally small so development of resistance may not be revealed. The kinetics of different routes of administration cannot be easily compared. Depending upon the organism and species this approach can have varying validity when compared to the human response. A method that can span the gap between placement of paper discs onto agar and the infusion of drug into an animal, precisely mimicking the human pharmacodynamic and pharmacodynamic profile would provide useful data. The capability to control both time and concentration of drug exposure to an organism is required.

\section{One-compartment module}

To address some of these issues and present the test organism with a dynamic concentration of drug that more closely mimics in vivo pharmacokinetics the one-compartment model was developed. This model consists of a central reservoir containing the organisms, a diluent reservoir and a waste reservoir. Peak concentrations of drug are added to the central reservoir. The elimination profile is mimicked by the addition of drug free diluent to the central reservoir and removal of an equal volume of drug (and organism) containing medium into the waste reservoir. The versatility of this model allows for the modeling of nearly any desired elimination half-life within the limits presented by the large volume of the central reservoir and the peristaltic pumps used to add and remove diluent. One disadvantage of the one-compartment model is the loss of organism during the dilution of the drug since the main parameter measured is the number of viable organisms. Another disadvantage is that only the elimination profile can be modeled, not the absorption profile. The size of the central reservoir restricts extremely rapid changes in drug concentration due to diffusional limitations. Another disadvantage is the biohazard presented while working with antibiotic resistant and pathogenic organisms in such an open system (Figure 3).

\section{Hollow fiber cartridge two-compartment models}

To address these shortcomings the two-compartment in vitro pharmacokinetic model utilizing hollow fiber bioreactors was developed. Hollow fiber bioreactors are modules containing thousand of hollow fibers; small tubular filters 200 microns in diameter. The fibers are sealed at each end so that liquid entering the ends of the

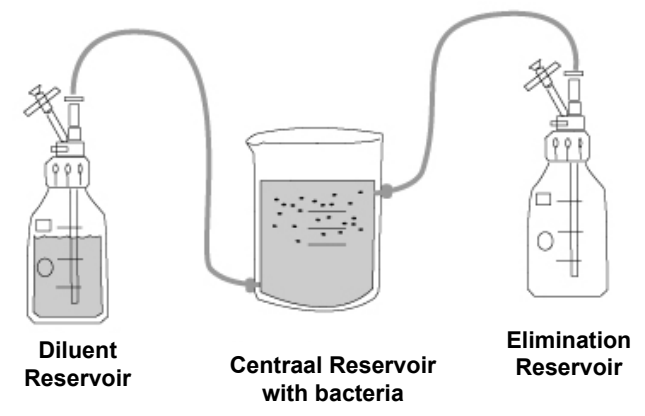

Figure 3: One compartment model. Volume remains constant but test organism number is not constrained and volume is relatively large. 
cartridge will necessarily go through the insides of the fibers. The pore size of the fibers is selected to retain the organisms while allowing drugs and other small molecule to freely cross the fiber. Bacteria or cells are inoculated on the outside of the fibers, trapped in the space called the extra-capillary space, or ECS. The ECS is defined by the space outside the fibers but within the cartridge housing. Medium from the central reservoir continuously recirculates through the inside of the fibers providing oxygenation and nutrition support. Small molecules such as drugs, glucose and metabolic waste products can easily cross the fiber while larger bacteria, cells and viruses cannot cross the fiber.

The design of the two-compartment model is quite similar to the one-compartment model except that the organism to be tested is confined within the small volume of the ECS $(20 \mathrm{mls})$, physically separated from the central reservoir by the semi-permeable membrane. The concentration of the drug in the central reservoir equilibrates rapidly with the medium in the ECS containing the organisms, which is relatively small in volume. The volume of the central reservoir can be adjusted to permit rapid changes in drug concentration (Figure 4).

Hollow fiber cartridges were first used by Zinner and Blaser [3] for bacterial testing in the 1980's and by Billelo et al. [4] for anti-HIV drugs in the 1990's. Hollow fiber cartridges offer the advantages of having an extremely high surface area to volume ratio, in excess of $150 \mathrm{~cm}^{2}$ per milliliter of volume, providing rapid and uniform distribution of the drug within the ECS. Several different types of hollow fiber polymers are commercially available to allow for compatibility with drugs of different chemistries.

There are specific advantages of the two-compartment hollow fiber infection model. The target bacteria are contained within a very small volume, $10-20 \mathrm{mls}$, so they are at a similar concentration to in vivo infections and the drug can equilibrate rapidly within the compartment. Representative samples can be taken easily without significantly affecting the bacteria population. Drug resistant, highly pathogenic and biohazardous organisms are safely contained in a sealed environment. Large numbers of organisms can be tested in one experiment so the emergence of drug resistance is easily quantified. Both absorption and elimination kinetics of the drug being testing can be precisely and independently controlled. The kinetics of multiple drugs can also be controlled so drug/drug interactions and combination therapies can readily be examined. The system is compact enough that multiple cartridges can be conveniently manipulated in a relatively small space (Figure 5).

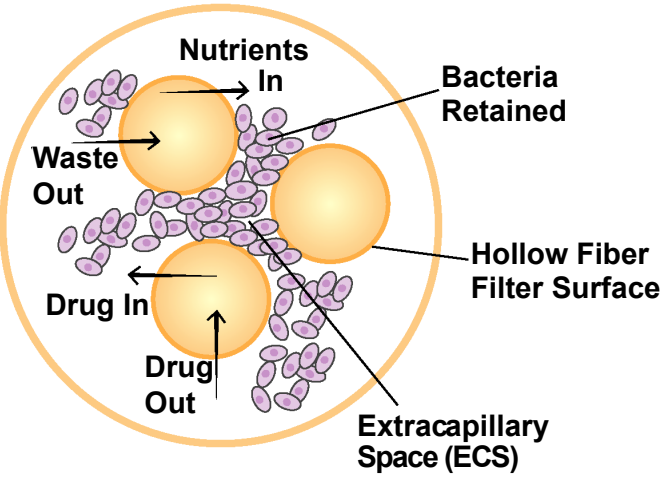

Figure 4: Cross-section of a hollow fiber cartridge. The test organism is retained in the small volume outside the fiber while nutrient broth and drug circulate through the insides of the fiber. Small molecules such as drugs can freely cross the fiber along with nutrients and waste products, bacteria and cells cannot cross the fiber.

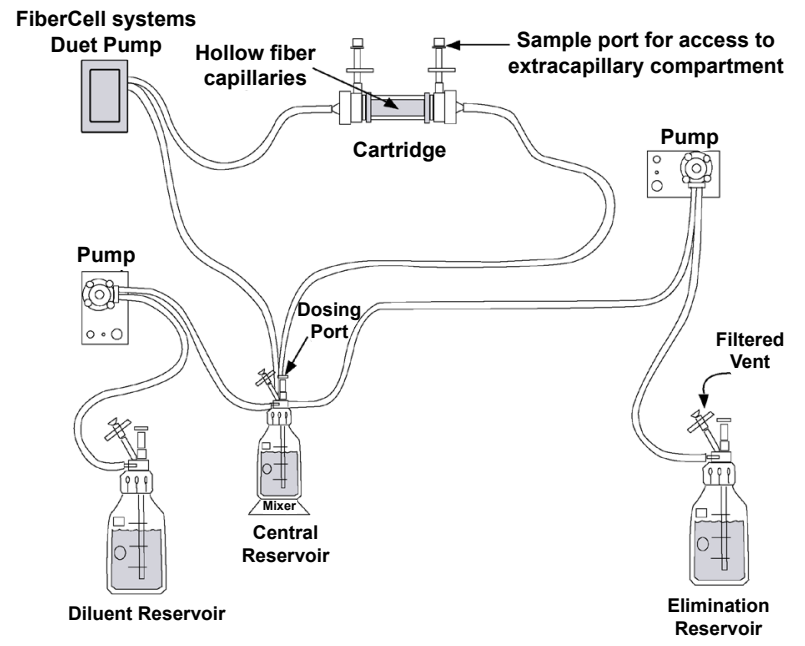

Figure 5: The hollow fiber two compartment model. Test organisms are retained in the hollow fiber cartridge. The central reservoir is continuously re-circulating the nutrient broth. Drug is added to the central reservoir and the elimination kinetics are controlled by the addition of diluent to the central reservoir. The volume in the central reservoir is kept constant.

The hollow fiber bioreactor based two-compartment model gained widespread acceptance as a result of the work by Drusano et al. with mammalian cells infected with HIV [5]. The testing of anti-viral agents is especially problematic as in most cases, such as HIV, there are no appropriate animal models and it can be difficult to culture sufficient cells to support viral growth. At the time of this work hollow fiber bioreactors were in common use for the production of monoclonal antibodies and recombinant proteins and had also been used for the production and growth of HIV. The two-compartment model utilizing hollow fiber bioreactor cartridges has been used for antibiotic testing against many organisms including Klebsiella pneumoniae [6], MRSA Staph aureus [7], pseudomonas aeruginosa [8] and tuberculosis [9]. The hollow fiber infection model has also been used time course dependant dose/response for anti-cancer agents as well [10]. Another area of great interest is in the determination of optimal antibiotic dosages for potentially weaponized organisms. In the event of a bioterrorist incident using modified organisms the hollow fiber method could rapidly determine the most effective antibiotics and their optimum dosage profiles. Lister and Wolter [11] demonstrated the power of the model for looking at antibiotic combination therapies. They demonstrated that the combination of levofloxacin and imipenem prevented the emergence of drug resistance from clinical isolates of Pseudomonas aeruginosa even when subpopulations resistant to both drugs are present. Drusano et al. [12] determined the relationship between garenoxacin exposure and emergence of quinalone resistant subpopulations and found that different targets for the area under the concentration-time curve over $24 \mathrm{~h} / \mathrm{MIC}$ ratio were required for different bacteria. The data from the hollow fiber two-compartment model can be critical for determining dosage profiles and drug combinations that can prevent the emergence of resistant strains. In the case of tuberculosis, data shows that ciprofloxacin and Isoniazid's antibiotic activity ceases not because of depletion of organisms in log growth phase but because of emerging resistance. These results could not have been found through any other type of study [10].

The two-compartment hollow fiber bioreactor system has also demonstrated distinct advantages when compared to previously used 
static and one-compartment models and can be used to determine optimum dosing schedules as well as revealing the mechanisms of resistance development. Historical in vitro methods for evaluating efficacy of antibiotics suffer from two fundamental shortcomings. The first is that antibiotic concentrations remain static; they are not varied in a dynamic fashion as they would be when administered in vivo. Flux in concentration of antibiotic should reflect the adsorption rate, bioavailability, volume of distribution and excretion rate. None of these parameters can be controlled using current methods. The second shortcoming is the number of organisms exposed to the drug is necessarily limited so mechanisms of resistance cannot be studied effectively. Hollow fiber bioreactors offer a more in vivo like way to model physiological processes. Bacteria can be grown at densities and numbers that reflect in vivo infection potential. The smaller volume provides more rapid equilibration of drug concentrations and a more uniform growth environment. This high-density culture also supports cell pathogen interactions such as virus infections and parasitic growth that more accurately reflect disease states. Therapeutic modalities utilizing antibiotic, anticancer and anti-parasitic agents depend not only upon a maximum tolerated dosage but the time course of administration, usually of multiple dosages. These can be easily studied in the hollow fiber infection model (Figure 6).

The hollow fiber infection model does present some challenges and disadvantages when compared to animal and other models. The cost of the hollow fiber cartridges is relatively high compared to the one compartment model but can be comparable to some types of animal studies. Careful experimental design is required to maximize the data collected. Proper controls need to be run. Volumes required for some high value drugs can be high. The hollow fiber infection model has no immune system nor serum for drug binding. Consequently, the drug exposure profile required for different levels of effect $(1,2$ or 3 Log kill, resistance suppression, etc.) are going to be somewhat biased high and must be recognized. An example is a study looking at Pseudomonas aeruginosa and a quinolone $[12,13]$. The hollow fiber infection model identified a free drug AUC/MIC ratio for suppression of emergence of resistance of about 190. Earlier this isolate was studied with levofloxacin, another quinolone, in a normal mouse thigh model (with intact immune system). Here [14] a total drug AUC/MIC ratio of 157 attained this same target. Levofloxacin is about $30 \%$ bound (and, therefore $70 \%$ free). Therefore, in the mouse, the free drug AUC/MIC

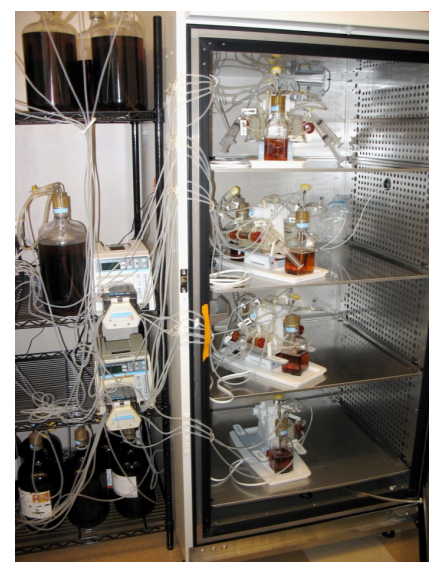

Figure 6: Typical laboratory set up of the hollow fiber two compartment model. The large volumes of diluent and the waste reservoirs are kept outside the incubator. Micro-processor controlled peristaltic pumps add and remove the diluent from the central reservoir inside the incubator. Sampling is performed via the side ports on the hollow fiber cartridge.

\section{Advantages of Hollow Fiber Bioreactor Cartridges vs. Static Methods}

- Dosage profiles can be controlled over time

- Mechanisms of resistance can be revealed

- Data is more clinically relevant

Advantages of Hollow Fiber Bioreactor Cartridges vs. the One-compartment Model

- Bacterial load remains constant

- Biohazardous organisms safely contained

- Both absorption and elimination curves can be modeled

- Rapid half lifes can be modeled

\section{Advantages of Hollow Fiber Bioreactor Cartridges vs. Animal Models}

- High bacterial and viral loads can be tested, dosage profiles that result in resistance will more likely be revealed

- Absorption, excretion and metabolic profiles can be more closely modeled on the human half-life

- Not all animals can be infected with the organism of interest

- Extreme doses can be modeled

- Combination therapies can be easily controlled and tested

- No animal testing protocols, restrictions

- Less expensive

- Closed, bio-safe system for pathogens

- Easier to test multiple pathogens with the same drug

- Larger $\mathrm{N}$ per experiment (cartridges in series)

- Bacteria, viruses, cancer and parasites can be tested

- Dosage and metabolic profiles can be more precisely controlled.

Table 1: Advantages of Hollow Fiber Bioreactor Cartridges.

ratio is 109.9. This means that hollow fiber identified an exposure about $72 \%$ higher (190 versus 110). This is a quite modest difference (MIC steps are $100 \%$ apart) but does need to be taken into account.

The other major issue is not so much a disadvantage as a reason to be careful. Many antibiotics and anti-virals of and organic nature can stick to one or the other fibers. This results in delays to or inability to achieve target concentrations in the peripheral chamber where the pathogen is. Consequently, it is critical to document the concentrationtime profile in the peripheral compartment and not just the central compartment. There are two fiber types available with very different drug binding profiles.

The Hollow Fiber Infection Model can provide useful data for antibiotic development and dosing. Hollow fiber technology offers higher levels of reproducible control of both concentration and time of drug exposure in complex growth, infection, treatment, and sampling regimens. This system permits a realistic simulation of in vivo drug effects in a dynamically controlled system providing data that more accurately reflects biological responses. They are fully disposable and provide a bio-safe environment for the potential testing of drug resistant, weaponized, or genetically modified organisms. The twocompartment model can be a cost effective supplement to the evaluation of clinical efficacy both for existing antibiotics and in the development process for new antibiotics as part of the submission process for FDA approval (Table 1).

\section{References}

1. EAGLE H (1954) The binding of penicillin in relation to its cytotoxic action. II The reactivity with penicillin of resistant variants of streptococci, pneumococci, and staphylococci. J Exp Med 100: 103-115.

2. White RL (2001) What in vitro models of infection can and cannot do Pharmacotherapy 21: 292S-301S

3. Blaser J, Zinner SH (1987) In vitro models for the study of antibiotic activities. Prog Drug Res 31: 349-381.

4. Bilello JA, Bauer G, Dudley MN, Cole GA, Drusano GL (1994) Effect of 2',3'-didehydro-3'-deoxythymidine in an in vitro hollow-fiber pharmacodynamic model system correlates with results of dose-ranging clinical studies. Antimicrob Agents Chemother 38: 1386-1391.

5. Preston SL, Piliero PJ, Bilello JA, Stein DS, Symonds WT, et al. (2003) In vitro- 
Citation: Cadwell JJS (2012) The Hollow Fiber Infection Model for Antimicrobial Pharmacodynamics and Pharmacokinetics. Adv Pharmacoepidem Drug Safety S1:007. doi:10.4172/2167-1052.S1-007

Page 5 of 5

In vivo Model for evaluating the Antiviral Activity of Amprenavir in combination with Ritonavir Administerred t 600 and 100 milligrams, Respectiviely, Every 12 hours. Antimicrobial agents and chemotherapy 47: 3393-3399.

6. Tam VH, Louie A, Deziel MR, Liu W, Drusano GL (2007) The relationship between Quinolone Exposures and Resistance Amplification is Characterized by an Inverted U: a New Paradigm for Optimizing Pharmacodynamics to counterselect Resistance. Antimicrob Agents Chemother 51: 744-747.

7. Osorio-Lozada A, Surapaneni S, Skiles GL, Subramanian R (2008) Biosynthesis of drug metabolites using microbes in hollow fiber cartridge reactors: case study of diclofenac metabolism by Actinoplanes species. Drug Metab Dispos 36: $234-240$

8. Lister PD (2006) The Role of Pharmacodynamic Research in the assessment of development of new antibacterial drugs. Biochem Pharmacol 71: 1057-1065.

9. Gumbo T, Louie A, Liu W, Ambrose PG, Bhavnani SM, et al. (2007) Isoniazid's bactericidal activity ceases because of the emergence of resistance, not depletion of Mycobacterium tuberculosis in the log phase of growth. J Infect Dis 195: 194-201.
10. Kirstein MN, Brundage RC, Moore MM, Williams BW, Hillman LA, et al. (2008) Pharmacodynamic Characterization of gemcitabine cytotoxicity in an in vitro cell cukture bioreactor systems. Cancer Chemother Pharmacol 61: 291-299.

11. Lister PD, Wolter DJ (2005) Levofloxacin-imipenem combination prevents the emergence of resistance among clinical isolates of Pseudomonas aeruginosa. Clin Infect Dis 40 Suppl 2: S105-114.

12. Drusano GL, Bilello PA, Symonds WT, Stein DS, McDowell J, et al. (2002) Pharmacodynamics of Abacavir in an In vitro Hollow-fiber Model System. Antimicrob Agents Chemother 46: 464-470.

13. Tam VH, Louie A, Deziel MR, Liu W, Leary R, et al. (2005) Bacterial-population responses to drug-selective pressure: examination of garenoxacin's effect on Pseudomonas aeruginosa. J Infect Dis 192: 420-428.

14. Tam VH, Kabbara S, Vo G, Schilling AN, Coyle EA (2006) Comparative pharmacodynamics of gentamicin against Staphylococcus aureus and Pseudomonas aeruginosa. Antimicrob Agents Chemother 50: 2626-2631.
This article was originally published in a special issue, Recent Trends in Pharmacokinetics/Pharmacodynamics handled by Editor(s). Dr. Richard L. Slaughter, Wayne State University, USA 